\title{
E. Frenk Die Anfänge der dermatologischen Universitätsklinik Stéphanie Liardet Lausanne an der Wende des 19. zum 20. Jahrhundert
}

\author{
The Beginnings of the University Department of Dermatology Lausanne at the \\ Turn of the 20th Century
}

\section{Zusammenfassung}

Die dermatologische Universitätsklinik Lausanne wurde 1891 als zweite eigenständige Schweizer Klinik gegründet. Geleitet von Emil Dind, beschäftigte sich die noch kleine Abteilung vor allem mit venerischen Krankheiten, wobei deren soziale Folgen im Vordergrund standen. Als Dinds Beitrag zur Therapie des Ekzems wurde erstmals die gute Wirkung von lokal appliziertem reinem Steinkohlenteer beschrieben.

\section{Abstract}

The University Department of Dermatology Lausanne was founded in 1891 and was the second independent unit created in Switzerland at the turn of the century. Directed by Emil Dind, it was mainly devoted to venereal diseases and their social consequences. Local application of pure coal tar was first promoted by Dind as an efficacious and well tolerated treatment for eczema.
Der Lehrstuhl und die damit verbundene eigenständige klinische Abteilung für venerische und kutane Krankheiten der Medizinischen Fakultät Lausanne wurden 1891 geschaffen. Es handelt sich um eine der ältesten dermatologischen Kliniken der Schweiz (Tab.1). Ihr erster Leiter und Professor war Emil Dind, der als erfolgreicher Politiker auch Wesentliches zur Gründung der medizinischen Fakultät und zur Gesetzgebung im Gesundheits- und Sozialwesen beigetragen hat. Die vorliegende Arbeit möchte zeigen, wie das im Verlauf des 19. Jahrhunderts entstandene Fachgebiet Dermatologie und Venerologie schon früh in die hiesige medizinische Versorgung eingeordnet wurde, und dies in einem von Landwirtschaft dominierten Kanton ohne großes urbanes Zentrum.

\section{Die Medizinische Fakultät der Universität Lausanne}

Die Gründung der Medizinischen Fakultät [1] war ein Resultat der im 19. Jahrhundert herrschenden Aufbruchstimmung. Nach dem Untergang der alten Schweizerischen Eidgenossenschaft im Jahre 1798 wurde das Waadtland 1803 als vollwertiger Kanton in den neuen schweizerischen Bundesstaat aufgenommen, nachdem es seit 1536 als Landvogtei von Bern verwaltet worden war.

Die noch von den Herren von Bern 1537 gegründete Akademie Lausanne zur Ausbildung von evangelisch reformierten Pfarrern wurde 1837 säkularisiert und durch eine philosophische und naturwissenschaftliche Fakultät ergänzt und schließlich durch die 1890 erfolgte Gründung der medizinischen Fakultät in eine unter der Verantwortung des neuen Kantons stehende Universität umgewandelt. In die zweite Hälfte des 19. Jahrhunderts fiel auch die Neuordnung des Spitalwesens. Das alte, 1282 gegründete und 1771 neuerbaute „Grand Hôpital“ war, wie in jener Zeit üblich, ein Asyl zur Unterbringung von Not leidenden und kranken Einwohnern und Pilgern gewesen. Es wurde 1873 und 1883 durch zwei moderne Zentren für Krankenpflege ersetzt, das psychiatrische Asyl Cery und das für nicht psychiatrische Patienten bestimmte Kantonsspital (Abb.1), in das dann auch die später errichtete dermatologische Klinik eingegliedert wurde. 


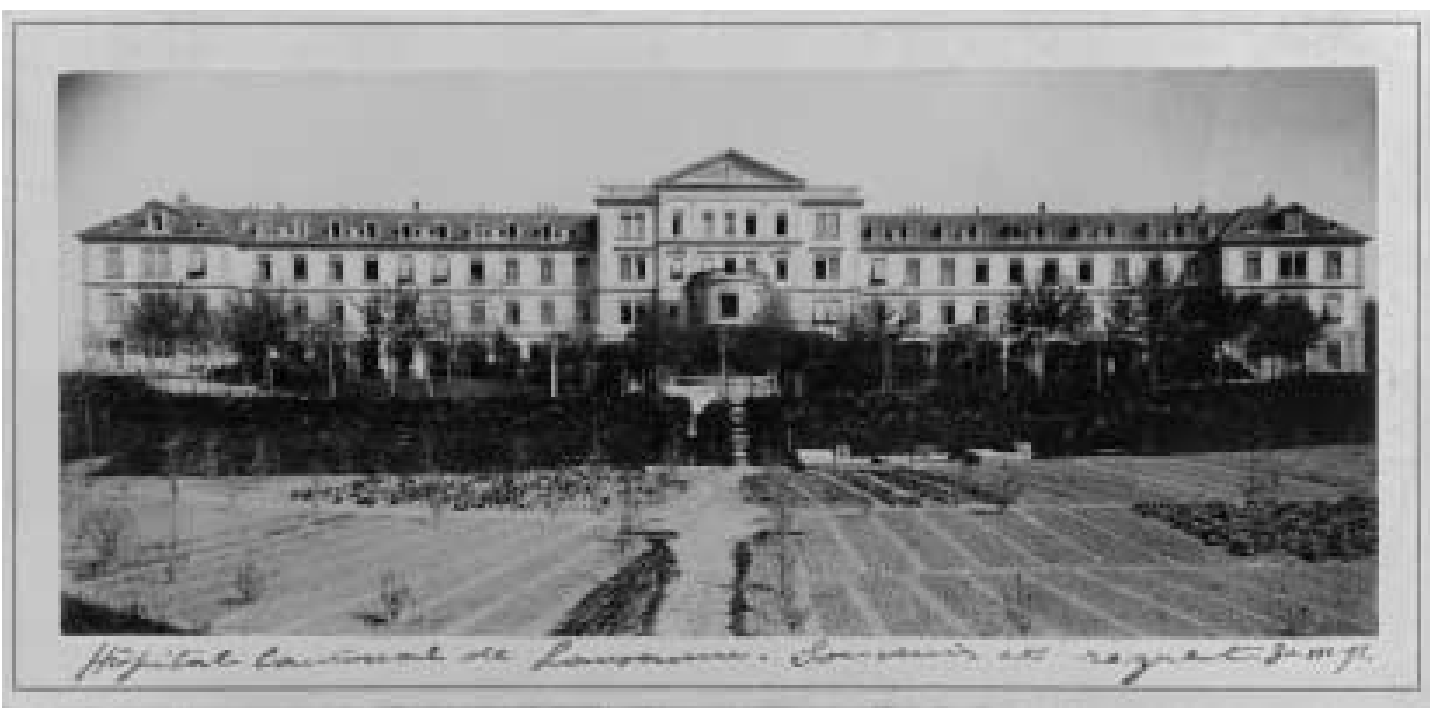

Abb. 1 Kantonsspital Lausanne zur Zeit der Gründung der Medizinischen Fakultät. Die dermato-venerologische Klinik befand sich im Erdgeschoss mit direktem Zugang zum Garten (Fotografie des Medizinhistorischen Instituts der Universität Lausanne).

Tab. 1 Dermatologische Universitätskliniken der Schweiz

\begin{tabular}{|c|c|c|}
\hline Universität & $\begin{array}{l}\text { Gründungsjahr } \\
\text { med. Fakultät/ } \\
\text { dermatol. Klinik }\end{array}$ & Vorläufer \\
\hline Basel & $1460 / 1914$ & $\begin{array}{l}\text { ab } 1760 \text { zeitweise Vorlesung über } \\
\text { Venerologie }\end{array}$ \\
\hline Bern & $1834 / 1892$ & $\begin{array}{l}\text { ab } 1834 \text { Vorlesungen über Syphilis im } \\
\text { „äußeren Krankenhaus“ }\end{array}$ \\
\hline Genf & $1876 / 1887$ & $\begin{array}{l}1559 \text { Akademie, ab } 1878 \text { theoretische } \\
\text { Vorlesungen über Dermatologie }\end{array}$ \\
\hline Lausanne & $1890 / 1891$ & 1537 Akademie \\
\hline Zürich & $1833 / 1916$ & 1788 privates med.-chir. Institut \\
\hline
\end{tabular}

Die Gründung der dermatologischen Universitätsklinik

Im ersten Fakultätsreglement von 1891 findet man unter den zu lehrenden medizinischen Wissensgebieten auch die venerischen und kutanen Krankheiten, was zur Errichtung eines Lehrstuhles mit zugehöriger klinischer Abteilung führte. Dass die venerischen Krankheiten an erster Stelle stehen, dürfte einerseits mit der zu jener Zeit sehr großen sozialen Bedeutung dieser Krankheiten zusammenhängen, andererseits vielleicht auch mit dem großen politischen Einfluss des damaligen Kantonsarztes Emil Dind, dem späteren Leiter dieser Klinik.

Die Dermatologie und Venerologie hat sich im Verlaufe der zweiten Hälfte des 19. Jahrhunderts als umschriebenes Fachgebiet vielerorts gut etabliert [2,3]. Ein wichtiger Anstoß zu dieser Entwicklung war das im Jahre 1776 publizierte Lehrbuch „Doctrina morbis cutaneis“ des Wiener Arztes Joseph Plenck (1733-1807), worin die Hautkrankheiten morphologisch geordnet wurden. In der Folge entstanden in Paris, London und Wien die ersten Zentren für Dermatologie, die Ausgangspunkte für die Errichtung von Lehrstühlen und Spezialkliniken in Europa und Nordamerika wurden. Regionale und nationale Fachgesellschaften wurden ab 1869 gegründet und der erste internationale Kongress fand 1889 in Paris statt. Einige Daten zur allgemeinen Entwicklung der Dermatologie und Venerologie sind in Tab. 2 summarisch dargestellt.
Tab. 2 Einige wichtige Daten zur Geschichte der Dermatologie und Venerologie des 19. Jahrhunderts

1776 „Doctrina de morbis cutaneis“, Joseph Plenck, Wien

1802 Jean Louis Alibert wird Arzt des Spitals St. Louis in Paris und beginnt dort seine Lehrtätigkeit mit Patientendemonstrationen im Freien

1808 "On cutaneous diseases“, Robert Willan, London

1813 „A practical synopsis of cutaneous diseases“, Thomas Bateman, London

1839 Nachweis von Pilzen bei Favus (Johann Lucas Schönlein, Zürich)

1841 Gründung der Blackfriar Klinik in London, der ersten dortigen Spezialklinik für Hautkrankheiten

1849 Errichtung von Lehrstühlen für Dermatologie (Ferdinand Hebra) und für Syphilis (Carl Ludwig Sigmund) in Wien

1873 Entdeckung des Mycobacterium leprae durch Armauer Hansen

1879 Nachweis der Gonokokken durch Albert Neisser

1889 Erster internationaler Kongress für Dermatologie in Paris

Die Lausanner Universitätsklinik fürr Dermatologie und Venerologie von 1891 bis 1925

Als erster Lehrstuhlinhaber, mit dem Titel eines außerordentlichen Professors, und Klinikleiter wurde Emil Dind ${ }^{1}$ (Abb. 2) gewählt. Seine Hauptanliegen waren die Geschlechtskrankheiten und deren soziale Folgen. Die Dermatologie betrieb er weitgehend als Autodidakt, da er keine eigentliche dermatologische Ausbildung in einer der zu jener Zeit in Blüte stehenden wenigen großen dermatologischen Schulen Europas durchlaufen hatte. Spätere Aufenthalte in Paris und Wien und Teilnahme an Kongressen ermöglichten Fortbildung und Erfahrungsaustausch.

Emil Dind wurde am 29.3.1855 als Sohn eines Landwirts in St. Cièrges im Kanton Waadt geboren. Sein Medizinstudium absolvierte er an den Universitäten Genf und Bern, wo er 1879 promovierte. 1880 eröffnete er in Cossonay eine ärztliche Praxis und 1885 wurde er zum Kantonsarzt gewählt. Als solcher reorganisierte er den kantonalen Sanitätsrat, die oberste Gesundheitsbe-

${ }^{1}$ Die Daten über Lebenslauf und politische Tätigkeit wurden den in der Presse erschienenen Nachrufen entnommen (Tribune de Lausanne, La Revue). 


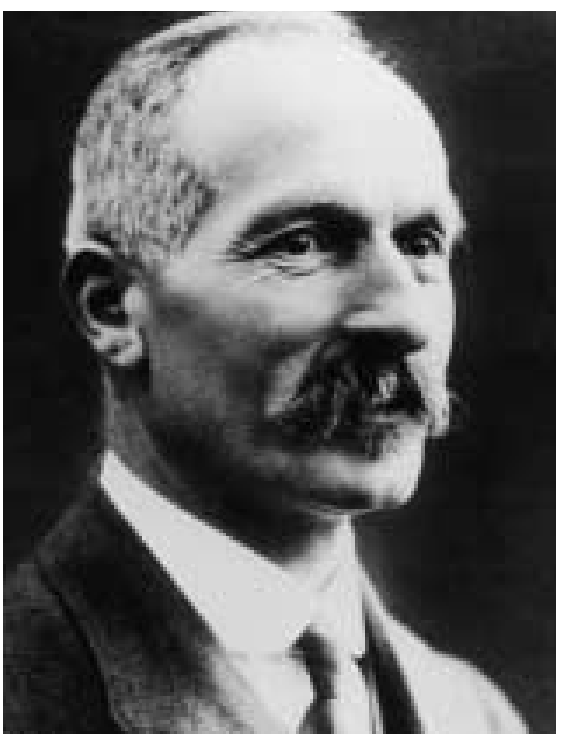

Abb. 2 Prof. Emil Dind, Leiter der dermato-venerologischen Klinik von 1891 bis 1925.

hörde des Kantons, und gab Anstoß zur Eröffnung eines Ambulatoriums („Dispensaire central“) für minderbemittelte Einwohner, aus dem später die Medizinische Poliklinik hervorging. Wichtig war auch seine Mitwirkung bei der 1887 ernannten Konsultativkommission der Kantonsregierung, welche die Grundlagen zur Gründung der medizinischen Fakultät ausarbeitete; sie bestand aus 6 Ärzten der beiden wichtigsten politischen Parteien des Kantons und tagte unter dem Vorsitz von Regierungsrat Eugen Ruffy, dem Vorsteher des Erziehungsdepartements [1].

Dind's Antrittsvorlesung [4] war, seinen Interessen entsprechend, im Wesentlichen der Syphilis und den damit verbundenen sozialen Problemen gewidmet; nur am Schluss folgten noch einige Worte zu den Hautkrankheiten, wobei er sich beklagt, dass die Lehrmeinungen darüber oft von Ort zu Ort verschieden sind und die verwendeten diagnostischen Begriffe in Paris und Wien häufig verschiedene Bedeutungen haben. Abschließend setzt er sich folgendes Ziel:

„De ceci on peut conclure que si tout n'est pas très clairement classé dans la nosologie cutanée, il n'y pas moins des faits acquis. C'est à les enseigner que nous vouerons nos soins. Laissant de côté les discussions spéculatives, nous nous appliquerons surtout à vous donner les notions diagnostiques et thérapeutiques les plus importantes. Grace à celles-ci, nous espérons vous mettre à même de faire face aux différentes situations que chaque médecin rencontre dans l'exercice de sa profession. “

Später diente Dind der Universität als Dekan der medizinischen Fakultät und 1904-1906 als Rektor. Als Klinikleiter befasste er sich, wie die Titel veröffentlichter Arbeiten zeigen, vor allem mit venerischen Erkrankungen (Tab.3). Bezeichnend für seine Ansichten war ein 1918 publizierter Artikel [5], in dem er sich gegen einen Vorschlag aussprach, venerische Patienten obligatorisch den Sanitätsbehörden zu melden. Es könne nicht Aufgabe des Arztes sein, Patienten, die freiwillig in seine Behandlung kämen, zu denunzieren. Ein solches Gesetz erschien ihm nicht durchsetzbar und somit unnötig und er verlangte dafür praktisch realisierbare Aufklärungskampagnen und das Errichten von speziellen Zentren, die sich den venerischen Patienten annehmen und, wenn nötig, auch gratis behandeln können.
Tab. 3 Emil Dind: veröffentlichte Arbeiten

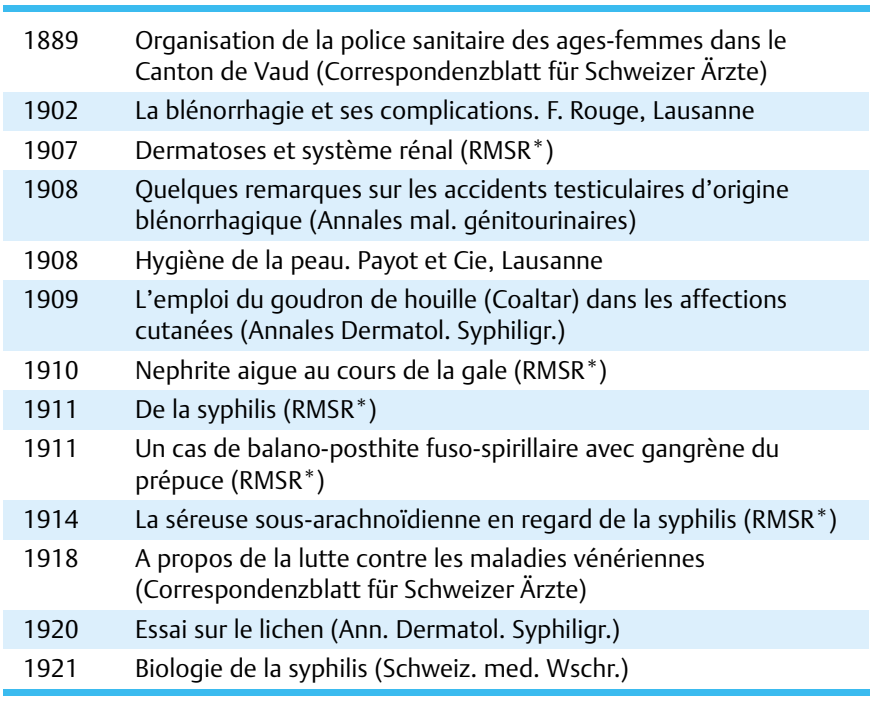

* RMSR : Revue médicale de la Suisse romande

Sein wichtigster Beitrag zur Dermatologie war sicher seine Arbeit über die Verwendung von Steinkohlenteer in der Ekzembehandlung [6]. Der billige halbflüssige Steinkohlenteer wird unverdünnt mit einem mit Watte umwickelten Holzstab in dünner Schicht appliziert und mit Talkpuder bestreut. Dind unterstrich die gute juckreizstillende und entzündungshemmende Wirkung, die weit besser sei als bei Teerapplikation in Fettsalben. Die mündliche Überlieferung (J. M. Paschoud, Lausanne) will es, dass Dind die Wirksamkeit von Steinkohlenteer dank seines Hundes entdeckte. Sein weißer Pudel, der sich seit Jahren wegen eines chronischen Ekzems kratzte, soll sich während Straßenarbeiten vor seinem Haus in dem dabei verwendeten Teer gewälzt haben. Nach dem ersten Schrecken der Familie Dind über das so verunstaltete Tier geschah das Wunder: der Pudel hörte auf, sich zu kratzen und als die Teerschicht langsam mit den Hautkrusten abfiel, war das Ekzem geheilt.

Im Jahre 1904 konnte Dind den Sanitätsrat von der Notwendigkeit überzeugen, am Kantonsspital eine Abteilung für Röntgentherapie einzurichten, die dann bis 1921 unter seiner Verantwortung blieb [7].

Bei der Klinikarbeit wurde Dind von einem Ober- und einem Assistenzarzt unterstützt. Eine einzige Laborantin genügte für die anfallenden Blut- und Urinuntersuchungen und die wenigen Hautbiopsien. Die Krankenpflege wurde von Diakonissen betreut.

Ein vor einigen Jahren in den Spitalarchiven gefundenes Manuskript [8] von Schwester Henriette Dufey, vermutlich eine Ansprache bei der Feier zu ihrem 35jährigen Dienstjubiläum, erzählt vom Klinikalltag. Sie arbeitete dort seit dem 22. Oktober 1915 , zuerst als „Badschwester der Frauenabteilung“; 1919 übernahm sie das Kinderzimmer und 1932 wurde sie Oberschwester. Ein paar übersetzte Abschnitte sollen einen Eindruck vom damaligen Leben in der Lausanner Klinik geben:

„Drei Krankensäle (der Frauenabteilung) beherbergten die Hautkrankheiten, einer die venerischen Fälle, die von sich aus zur Be- 
handlung kamen, und ein weiterer Prostituierte sowie Inhaftierte. Eine ganz kleine Untersuchungskabine, nur rudimentär ausgestattet, in der Ecke dieses letzten Saales diente als Behandlungslokal für die venerischen Patienten beider Säle und für ambulante Patienten. Die Insassen benachbarter Betten hatten somit einen ersten Logenplatz, um zu hören, was in der Kabine vorging. Die Patienten wurden jeden Montag untersucht. Bei Gonorrhö wurde mit Zinkchlorid irrigiert und Protargol instilliert“...

„Professor Dind operierte auch Prostatapatienten. Trotz unperfekten Sterilisierungsmethoden kann ich von keinem einzigen Fall von Infektion berichten...Der Professor begnügte sich, über dem Arbeitsmantel eine Schürze zu tragen, die Operationsschwester arbeitete im schwarzen Rock. Die sterilen Operationstücher wurden in einer Küvette gekocht, gewrungen und dann auf das Operationsfeld gelegt. Nach beendeter Operation beeilte sich Herr Dind, die Prostata zu wiegen, da er immer an ihrem Gewicht interessiert war.“...

„Herr Dind war ein ausgezeichneter Professor und bei seinen Studenten sehr beliebt. Er war auch ein großer Freund der Politik; er war Ständerat in Bern und seine Worte mussten Wunder wirken, denn er war ein ausgezeichneter Redner. Wenn er in Bern war, genoss die Klinik Momente der Entspannung, denn er war manchmal ein strenger Chef und konnte bei seinen Untergebenen Furcht erwecken“...

1897 wurde Dind in den Gemeinderat der Stadt Lausanne gewählt. Von 1901 bis 1921 war er Mitglied des Großen Rates (Legislative des Kantons), 1911/12 als dessen Präsident. Dort setzte er sich für Schulen, soziale Dienste und eine kantonale Rentenversicherung ein. Höhepunkt seiner politischen Laufbahn war seine 1917 erfolgte Wahl in den Ständerat, dem er als Vertreter des Kantons Waadt bis 1931 angehörte. Er war Mitglied der wichtigen Finanzdelegation, weiter befasste er sich mit der Einführung eines einheitlichen schweizerischen Strafrechts, dem Beamtenstatut und dem Gesetz zur Tuberkulosebekämpfung.

Dind trat 1925 als Professor und Klinikleiter in den Ruhestand. Sein Nachfolger wurde Edwin Ramel, der in der damals wissenschaftlich sehr aktiven, von Bruno Bloch geleiteten Zürcher Klinik zum Dermatologen ausgebildet worden war. Damit wurde auch der Übergang eingeleitet von der vorwiegend venerologisch orientierten Spezialklinik zur eigentlich dermatologischen $\mathrm{Ab}$ teilung. Emil Dind starb im Alter von 77 Jahren am 11. September 1932, 2 Jahre nach einem invalidierenden Hirnschlag, von dem er sich nicht mehr erholte.

\section{Literatur}

${ }^{1}$ Saudan G. La médecine à Lausanne. Denges: Editions du Verseau, 1991 ${ }^{2}$ Crissey JT, Parish LC. The dermatology and syphilology of the nineteenth century. New York: Praeger Publ, 1981

${ }^{3}$ Tilles G. La naissance de la dermatologie (1776-1880). Paris: Editions R. Dacosta, 1989

${ }^{4}$ Dind E. Leçon d'ouverture. In: Discours d'installations et leçons inaugurales. Lausanne: Editions de l'Université, 1894

${ }^{5}$ Dind E. A propos de la lutte contre les maladies vénériennes. Correspondenzblatt für Schweizer Ärzte 1818; 18: 572 - 574

${ }^{6}$ Dind E. L'emploi du goudron de houille (coaltar) dans les affections cutanées. Ann dermatol syphiligr 1909; 10: 170-178

${ }^{7}$ Terrier G, Calinon D, Ostini S. Histoire des débuts de la radiologie à Lausanne (1896-1921). Rev méd Suisse rom 1996; 116: 1001 - 1008

${ }^{8}$ Frenk E. Souvenirs de Soeur Henriette Dufey. Dermatologica Helvetica 1998; 4: 8-9

\section{Hinweis: \\ Die Festschrift zum 70. Geburtstag für Herrn Prof. Dr. E. G. Jung wird in Heft 5 mit den folgenden Beiträgen fortgesetzt:}

Selektive Cyclooxygenase II Hemmer bei Patienten mit pseudoallergischen Reaktionen auf nichtsteroidale Antiphlogistika

C. Termeer, E. Schöpf, J. C. Simon

Die labialen und oralen Pigmentflecken des Peutz-Jeghers-Syndroms W. Back, S. Loff, E. Dippel, W. Friedl, D. Jenne, U. Bleyl

Sjögren-Larsson-Syndrom mit reduzierter Fettaldehyddehydrogenase-Aktivität bei einem Kollodiumbaby und konsanguinen Eltern Christiane Bayerl, M. Kurzen, I. Treiss, I. Haußler

Behandlung der Schuppenflechte mit Interferenzstrom Elektromagnetische Therapie auf neuen Wegen H. Dertinger, K.-F. Weibezahn

Therapiefraktäres Ulcus cruris leukozytoklastisch-vaskulitischer Genese im Rahmen eines hereditären Thrombophilie-Syndroms T. Ermuth, S. Johnsen, W. Vanscheidt 